\title{
Leguminosas nativas: estrategias adaptativas y capacidad para la fijación biológica de nitrógeno. Implicancia ecológica
}

\author{
Native legumes: adaptive strategies and capacity for biological nitrogen fixation. \\ Ecological implication
}

\author{
Luciana Bianco*1 y Ana M. Cenzano ${ }^{2}$
}

\begin{abstract}
RESUMEN
El calentamiento global, la pérdida de la biodiversidad y la desertificación constituyen los principales problemas que debe afrontar el planeta. Muchas leguminosas nativas son componentes importantes de nuestros ecosistemas áridos y semiáridos. La mayoría presentan adaptaciones morfofisiológicas frente al estrés hídrico y capacidad para la fijación biológica de nitrógeno, lo que las convierte en en especies promisorias de estos ecosistemas desérticos. Los objetivos de esta revisión son: a) Describir la situación actual de la desertificación y las principales estrategias adaptativas de las leguminosas nativas de zonas áridas y semiáridas, y b) Analizar la diversidad de microorganismos que nodulan las leguminosas nativas y su importancia en la fijación biológica de nitrógeno. Esta revisión pone de manifiesto que las leguminosas nativas y sus simbiontes representan un germoplasma único que puede adoptarse para la restauración de los ecosistemas degradados, en el mantenimiento de la biodiversidad y para mitigar los efectos del cambio climático. Además, los trabajos publicados muestran grandes avances en la taxonomía rizobiana y en estudios de modificaciones morfoanatómicas a nivel de sistema radical que les proporcionan a las especies tolerancia a distintos factores de estrés como sequía, salinidad, suelos con $\mathrm{pH}$ alcalino y suelos de baja fertilidad. Aún hay muchas especies de leguminosas nativas con caracteres adaptativos y capacidad de establecer simbiosis con los microorganismos del suelo que no han sido investigadas. Por ello sería de gran importancia continuar con los estudios a fin de aplicar estrategias de manejo y conservación de especies de interés para prevenir el avance de la desertificación en regiones áridas y semiáridas.
\end{abstract}

Palabras clave: desertificación, leguminosas nativas, adaptaciones morfofisiológicas, fijación biológica de nitrógeno

\begin{abstract}
Global warming, loss of biodiversity and desertification are main problems that planet must face. Many native legumes are important components of our arid and semi-arid ecosystems. Most of them present morpho-physiological adaptations to water stress and capacity for biological fixation of nitrogen, which constitutes them as promising species of these desert ecosystems. The objectives of this review are: a) to describe the current situation of desertification and the main adaptive strategies of native legumes in arid and semi-arid zones, and $b$ ) to analyze the diversity of microorganisms that nodulate native legumes and their importance in fixing biological nitrogen. This review shows that native legumes and their symbionts represent a unique germplasm that can be adopted for the restoration of degraded ecosystems, in the maintenance of biodiversity and to mitigate the effects of climate change. In addition, the published works show great advances in the rhizobial taxonomy and studies of morpho-anatomical modifications of radical system provides the species tolerance to different stress factors such as drought, salinity, soils with alkaline pH and low soils fertility. There are still many species of native legumes with adaptive characters and ability to establish symbiosis with soil microorganisms that have not been investigated. Therefore, it would be very important to continue with studies in order to apply management strategies and conservation of species of interest to prevent the advance of desertification in arid and semi-arid regions.
\end{abstract}

Keywords: desertification, native legumes, morpho-physiological adaptations, biological fixation of nitrogen

1 Laboratorio de Morfología Vegetal, Facultad de Agronomía y Veterinaria, Universidad Nacional de Río Cuarto, Ruta Nac. 36 - km 601. 5800, Río Cuarto, Provincia de Córdoba, Argentina.

2 Laboratorio de Ecofisiología y Bioquímica Vegetal. Instituto Patagónico para el Estudio de los Ecosistemas Continentales (IPEEC). Centro Científico Tecnológico CONICET - Centro Nacional Patagónico (CCT CONICET-CENPAT). Boulevard Brown 2915. 9120, Puerto Madryn, Chubut.

* Autor corresponsal: 1bianco@ayv.unrc.edu.ar.

Fecha de recepción: 30 noviembre, 2017.

Fecha de aceptación: 17 junio, 2018.

DOI: 


\section{Introducción}

En el planeta se registran tres grandes problemas ambientales que pueden interactuar entre sí, como son el calentamiento global, la pérdida de la biodiversidad y la desertificación. La desertificación definida como la degradación de las tierras secas, correspondientes a las regiones áridas, semiáridas y subhúmedas secas, es un problema socioeconómico de alcance global. Este proceso es la resultante de las variaciones climáticas y de las actividades humanas (Abraham, 2002).

El clima juega un papel importante en la desertificación, pero no es determinante del proceso. Entre los factores climáticos que influyen se pueden destacar la elevada temperatura, el elevado nivel de radiación solar, la baja humedad relativa, la elevada evaporación y la escasez de precipitaciones (Delgado-Baquerizo et al., 2013).

Además, las actividades humanas han incrementado la degradación del suelo en todo el mundo. Las que más han influido en el avance de la desertificación son el pastoreo excesivo, la deforestación, el inadecuado manejo del recurso hídrico, el uso inapropiado de fertilizantes y la expansión de áreas agrícolas hacia áreas frágiles, entre otras (Granados-Sánchez et al., 2012).

En las regiones áridas y semiáridas el pastoreo excesivo y la deforestación destruyen la cubierta vegetal provocando una reducción a largo plazo de la cantidad y la diversidad de las especies nativas. En consecuencia, surgen problemas de erosión hídrica y eólica que decapitan los estratos fértiles del suelo. Las prácticas agrícolas no sustentables llevan también a que los suelos sean deficientes en nutrientes, con elevada salinización, compactación y acumulación de sustancias tóxicas. Todos estos factores causan una menor productividad de las tierras y menor calidad de vida de las personas y en última instancia llevan a condiciones de pobreza y abandono de estas zonas (Abraham, 2002; Granados-Sánchez et al., 2012).

Actualmente la desertificación es uno de los mayores desafíos ambientales que enfrenta la sociedad humana. Comprender cómo los distintos factores influyen sobre este proceso, permitirá predecir la respuesta de los ecosistemas a los cambios ambientales minimizando el daño. Por otro lado, es de suma importancia la rehabilitación de los suelos degradados para que puedan destinarse a otros usos, tales como la producción de cultivos, forrajes, leña o simplemente para fines ambientales y de conservación (Delgado-Baquerizo et al., 2013).

Muchas leguminosas nativas son componentes importantes de nuestros ecosistemas áridos y semiáridos. La mayoría de estas especies presentan adaptaciones morfofisiológicas frente al estrés hídrico y salino. En suelos pobres de nitrógeno sus raíces se asocian con los rizobios del suelo, dando origen a estructuras especializadas denominadas nódulos, donde se produce la fijación biológica de nitrógeno (FBN) atmosférico. Estas características favorecen la supervivencia y productividad de las leguminosas en zonas donde otras plantas no pueden crecer ni establecerse, por lo que se constituyen en especies promisorias de ecosistemas desérticos (Sprent y Parsons, 2000).

En estos últimos años, ante la necesidad de responder a las amenazas de un cambio climático y un aumento de la desertificación, se despertó un mayor interés, por parte de los investigadores, en comprender cómo ciertas leguminosas y los simbiontes asociados son capaces de sobrevivir y fijar nitrógeno atmosférico en un ambiente de condiciones adversas. Por otro lado, se ha determinado que las leguminosas nativas podrían cumplir un rol importante en la conservación y reforestación de zonas desérticas (Sprent y Parsons, 2000). En Argentina y en la mayoría de los países de América Latina hay muchas especies de leguminosas nativas con capacidad de adaptación y de fijar nitrógeno atmosférico que no han sido estudiadas aún en su hábitat natural, y que podrían ser utilizadas en zonas del país propensas a la desertificación. Por todo lo expuesto, los objetivos de esta revisión son: a) Describir la situación actual de la desertificación y las principales estrategias adaptativas de las leguminosas nativas de zonas áridas y semiáridas, y b) Analizar la diversidad de microorganismos que nodulan las leguminosas nativas y su importancia en la fijación biológica de nitrógeno.

\section{Situación actual de la desertificación- Importancia de las leguminosas nativas}

La sequía es considerada como uno de los mayores desastres naturales del mundo. Ejerce un importante efecto negativo sobre la producción agrícola y también es la causante de impactos adversos sobre el medioambiente. A nivel mundial, más de 6.100 millones de hectáreas (ha) son consideradas zonas áridas y semiáridas, lo cual 
representa un porcentaje del $40 \%$ de la superficie del planeta. Esto afecta directamente el bienestar y el futuro de una sexta parte de la población mundial (Granados-Sánchez et al., 2012). En América Latina y el Caribe, de 20 millones de $\mathrm{km}^{2}$ que conforman la superficie total, cerca de 5 millones de $\mathrm{km}^{2}(26 \%$ del territorio) son tierras secas. Por esta razón se han aumentado los estudios sobre desertificación en toda esta región. En América Latina, Argentina es el país con mayor superficie árida, semiárida y subhúmeda seca ( $75 \%$ de su superficie) y el $81,5 \%$ del territorio correspondiente a las zonas secas se encuentra afectado por la desertificación. De la superficie total del país (alrededor de 224.000.000 ha), 60.000.000 ha han sufrido erosión y se calcula que por año 650.000 ha son afectadas por la erosión en diversos grados. Estos procesos se agravan en las zonas áridas y semiáridas que constituyen un alto porcentaje del territorio argentino. Otro aspecto importante es que la mayor parte de la superficie de este país (el 80\%) se destina a actividades de agricultura, ganadería y silvicultura, por lo que resulta prioritario detener el avance de las condiciones desérticas hacia otros suelos productivos (Abraham, 2002).

En la actualidad se están propiciando planes nacionales de lucha contra la desertificación, cuyas principales actividades tienden a la prevención, reducción, rehabilitación y recuperación de las tierras desertificadas (Abraham, 2002). Muchas propuestas surgen para solucionar esta problemática, entre ellas, una muy importante es disminuir la pérdida de la biodiversidad y la explotación sustentable de especies nativas. Para ello es necesario identificar y preservar las especies que habitan las zonas áridas y semiáridas. Entre las especies nativas que viven en estas áreas se encuentran aquellas pertenecientes a la familia de las leguminosas, la cual es muy diversa y cuenta con aproximadamente 19.000 especies y 650 géneros distribuidos en los distintos ecosistemas del mundo (Lewis et al., 2005). Muchas de ellas tienen gran importancia a nivel ecológico y económico, y se utilizan para diversos fines, tales como alimentos, fibra, leña, madera, ornamentales y productos farmacológicos (Sprent y Parsons, 2000). Teniendo en cuenta todos los atributos mencionados, continúan los esfuerzos para investigar nuevas especies nativas que presenten potencial para uso comercial y a su vez estén adaptadas a ambientes marginales (Lewis et al., 2005; Villagra et al., 2010).

\section{Estrategias adaptativas de las leguminosas nativas de zonas áridas y semiáridas}

La diversidad y la productividad de las zonas áridas y semiáridas dependen de la interacción entre las adaptaciones desarrolladas por los organismos y las limitaciones ambientales que presenta cada región en particular (Villagra et al., 2010). El crecimiento y desarrollo de una planta depende del ambiente donde vive. Muchas veces, ante condiciones ambientales variables, las plantas sufren diferentes tipos de estrés a lo largo de su ciclo de vida. Sin embargo, algunas presentan mayor plasticidad adaptativa, la cual se define como la capacidad global de un organismo de funcionar apropiadamente en respuesta a un estímulo ambiental. Estos cambios fenotípicos que ocurren durante el desarrollo vegetal tienen como objetivo principal el éxito reproductivo (Sultan, 2003).

Se ha observado que las leguminosas nativas son capaces de adaptarse a condiciones ambientales estresantes (Villagra et al., 2010; Cenzano et al., 2016). Sus adaptaciones abarcan diversas características morfológicas, anatómicas, bioquímicas y fisiológicas que constituyen estrategias para escapar, evitarotolerar el estrés hídrico y la salinidad, entre otros factores. En la Tabla 1 se presentan las principales características morfológicas, anatómicas y ecofisiológicas para resistir a la sequía en leguminosas.

Las especies que son capaces de escapar a la sequía se caracterizan por completar su ciclo de vida antes de un período de estrés hídrico (Levitt, 1980). Esta estrategia de adaptación la presentan Indigofera cordifolia, Indigofera linnaei, Indigofera sessiliflora pertenecientes a la subfamilia Mimosoideae y Alysicarpus vaginalis y Rhynchosia aurea pertenecientes a la subfamilia Papilionoideae (Gehlot et al., 2012). Las especies que evitan la sequía previenen la deshidratación celular (Levitt, 1980) y entre ellas se encuentra Acacia caven perteneciente a la subfamilia Mimosoideae (Raab et al., 2015). Finalmente, las especies que toleran la sequía poseen mecanismos que les permiten resistir la deshidratación celular (Levitt, 1980). Esta estrategia la presentan especies como Acacia jacquemontii, Mimosa hamata, Prosopis cineraria pertenecientes a la subfamilia Mimosoideae, y Crotalaria burhia, Rhynchosia minima, Tephrosia purpurea, Tephrosia uniflora, Tephrosia villosa pertenecientes a la subfamilia Papilionoideae (Gehlot et al., 2012). 
Tabla 1: Estrategias adaptativas de las leguminosas nativas de zonas áridas y semiáridas.

\begin{tabular}{|c|c|c|c|}
\hline $\begin{array}{l}\text { Estrategias de resistencia a } \\
\text { la sequía en leguminosas }\end{array}$ & Escape & Evitación & Tolerancia \\
\hline $\begin{array}{l}\text { Características } \\
\text { morfológicas y anatómicas }\end{array}$ & $\begin{array}{l}\text { - Algunas poseen hábito de } \\
\text { crecimiento postrado } \\
\text { - Ciclo de vida corto ( } 2 \text { o } 3 \\
\text { meses), anuales o perennes } \\
\text { - Tallo, hojas y legumbres } \\
\text { pilosas } \\
\text { - Sistema radical ramificado } \\
\text { - Capaces de adelantar su } \\
\text { floración y reproducción } \\
\text { antes de los períodos de } \\
\text { intensa sequía }\end{array}$ & $\begin{array}{l}\text { - Sistema radical superficial } \\
\text { - Raíces suberificadas } \\
\text { - Hojas deciduas, de corta } \\
\text { duración, pequeñas, con } \\
\text { movimientos paraheliotrópicos } \\
\text { (que reducen absorción de } \\
\text { radiación solar) }\end{array}$ & $\begin{array}{l}\text { - Algunas poseen hábito de crecimiento } \\
\text { postrado } \\
\text { - Sistema radical profundo } \\
\text { - Alta relación biomasa radical/biomasa } \\
\text { aérea } \\
\text { - Presencia de espinas } \\
\text { - Tallos fotosintéticos gruesos y ramificados } \\
\text { - Hojas siempre verdes, de larga duración, } \\
\text { pequeñas, duras, a veces dispersas, con } \\
\text { ceras epicuticulares y pilosas } \\
\text { - Corteza gruesa en tallo y raíz } \\
\text { - Presencia de cutícula en tallo y hojas } \\
\text { - Gran cantidad de fibras en tallo y hojas } \\
\text { - Legumbre pilosas y semillas con } \\
\text { tegumento seminal duro }\end{array}$ \\
\hline $\begin{array}{l}\text { Características eco- } \\
\text { fisiológicas }\end{array}$ & $\begin{array}{l}\text { - Sensibles a períodos } \\
\text { de interpulsos de } \\
\text { precipitaciones, respondiendo } \\
\text { más al incremento en la } \\
\text { disponibilidad de agua }\end{array}$ & $\begin{array}{l}\text { - Elevada tasa de crecimiento } \\
\text { - Alto potencial para captación } \\
\text { de recursos } \\
\text { - Elevada actividad metabólica } \\
\text { - Elevada conductancia } \\
\text { estomática, tasa fotosintética } \\
\text { y concentración de N en sus } \\
\text { hojas } \\
\text { - Baja inversión en } \\
\text { compuestos secundarios }\end{array}$ & $\begin{array}{l}\text { - Baja tasa de crecimiento } \\
\text { - Alto potencial para conservación de } \\
\text { recursos } \\
\text { - Elevada concentración de sustancias } \\
\text { osmoprotectoras, tales como prolina. } \\
\text { - Capaces de reducir la pérdida de agua a } \\
\text { través del cierre de estomas y mantener la } \\
\text { turgencia celular mediante ajuste osmótico } \\
\text { - Acumulación de ácido abscísico en } \\
\text { respuesta a la sequía } \\
\text { - Elevada inversión en compuestos } \\
\text { secundarios } \\
\text { - Exclusión de iones tóxicos de la parte } \\
\text { aérea, traslocación de fotoasimilados a } \\
\text { órganos subterráneos, para incrementar el } \\
\text { crecimiento del sistema radical y asegurar } \\
\text { una mayor disponibilidad de agua y } \\
\text { nutrientes }\end{array}$ \\
\hline Referencias & $\begin{array}{l}\text { (Levitt, 1980; Gehlot et al., } \\
\text { 2012; Cenzano et al., 2016) }\end{array}$ & $\begin{array}{l}\text { (Raab et al., 2015: Levitt, } \\
\text { 1980; Cenzano et al., 2016) }\end{array}$ & $\begin{array}{l}\text { (Levitt, 1980; Gehlot et al., 2012; Cenzano } \\
\text { et al., 2016) }\end{array}$ \\
\hline
\end{tabular}

Además de las estrategias mencionadas, muchas leguminosas nativas presentan otros tipos de modificaciones morfoanatómicas importantes a nivel de su sistema radical, que no solo les permiten adaptarse a ambientes extremos alterando el $\mathrm{pH}$ del suelo, sino también explotar con más eficiencia los nutrientes limitantes (Tola, 2009). Entre las especies de leguminosas más conocidas se encuentran Hedysarum coronarium, Hedysarum glomeratum, Hedysarum spinosissimum, Lupinus albus, Lupinus cosentinii y Lupinus luteus. Hedysarum coronarium es una leguminosa nativa del Mediterráneo, que se destaca por su tolerancia a distintos factores de estrés como sequía, salinidad y suelos con $\mathrm{pH}$ alcalino. Se adapta a vivir en áreas marginales y desiertos, y es considerada beneficiosa debido a sus propiedades antierosión conferidas por su vigoroso sistema radical (Tola, 2009). Cuando crece en suelos alcalinos desarrolla raíces laterales modificadas cortas, aplanadas y curvas en forma de pala. Estas se forman debido a una distribución asimétrica del volumen celular de las células corticales, que son más grandes 
en el lado convexo y están constituidas por parénquima reservante y en el lado cóncavo poseen gran densidad de pelos radicales que aumentan la superficie de contacto y mejoran la absorción de nutrientes limitantes (Tola, 2009). Estas raíces denominadas shovel roots son capaces de remover el calcio del suelo, absorberlo y acumularlo intracelularmente en formas de cristales de carbonato de calcio $\left(\mathrm{CaCO}_{3}\right)$ incidiendo en el $\mathrm{pH}$. Una acidificación del suelo puede conducir no solamente a aumentar la disponibilidad del hierro, sino también a una mejor captación de todos los nutrientes, los cuales estaban afectados por las condiciones alcalinas. Además, esta especie es capaz de establecer simbiosis con los rizobios y de formar micorrizas (Tola, 2009). En especies de Lupinus y Acacia se encontraron otro tipo de modificaciones denominadas cluster roots, como respuesta a hábitats con suelos de baja fertilidad y funcionalmente asociadas con una intensa movilización de nutrientes (P, Fe, Zn, Mn) por cambios químicos que producen en la rizósfera. Consisten en agrupamientos de raíces laterales de crecimiento limitado. Cada una mide aproximadamente 1-3 cm de longitud, están cubiertas por pelos radicales y exudan grandes cantidades de compuestos fenólicos, citratos y malatos. Se originan del periciclo opuesto a los polos de protoxilema y tienen una vida efímera. Si bien shovel roots y cluster roots son distintos tipos de modificaciones morfoanatómicas a nivel del sistema radical, ambas cumplen un rol importante en la solubilización de nutrientes limitantes (Tola, 2009).

Otras especies como Robinia pseudoacacia y Medicago lupulina desarrollan raíces contráctiles que poseen una serie de funciones combinadas como contracción, reserva de sustancias y propagación que regulan la posición de las yemas en el suelo (Pütz, 1996).

\section{Diversidad de microorganismos que nodulan las leguminosas nativas y su importancia en la FBN}

El éxito de las leguminosas nativas no sólo es atribuido a que poseen adaptaciones importantes frente a distintas condiciones de estrés, sino también a su capacidad para fijar nitrógeno $\mathrm{N}$ atmosférico (Sprent y Parsons, 2000). Las interacciones simbióticas con los microorganismos del suelo incrementan su plasticidad adaptativa. En particular, esta familia puede ser nodulada por una gran diversidad de bacterias (Weir, 2016).

\section{Diversidad de microorganismos que nodulan las leguminosas nativas.}

En los últimos años, el análisis de la diversidad de rizobios que nodulan las leguminosas nativas ha aportado resultados interesantes en cuanto a la taxonomía rizobiana. En la actualidad se incluyen 13 géneros en los cuales hay más de $98 \alpha$-Proteobacterias y $\beta$-Proteobacterias que son capaces de participar en la simbiosis. La mayoría de estas especies bacterianas se encuentran en la familia Rhizobiaceae, clase $\alpha$-Proteobacterias. Se destacan los géneros Rhizobium, Mesorhizobium, Ensifer y Bradyrhizobium. Sin embargo, estudios realizados recientemente han demostrado que existen otros géneros además de estos, los cuales han surgido a través de la transferencia lateral de genes simbióticos (Weir, 2016). Entre ellos se encuentran Aminobacter, Azorhizobium, Blastobacter, Devosia, Methylobacterium, Microvirga, Ochrobactrum, Phyllobacterium y Shinella correspondientes a la clase $\alpha$-Proteobacterias y Burkholderia y Cupriavidus correspondientes a la clase $\beta$-Proteobacterias. Estos géneros han adquirido importancia para la agricultura debido a su capacidad de fijar considerable cantidad de nitrógeno atmosférico en simbiosis con las leguminosas (Weir, 2016).

La diversidad de los rizobios está relacionada con la distribución geográfica de las leguminosas. En algunos casos, existe correlación entre la especie de rizobio y la región ecológica. Las interacciones entre la bacteria, la planta huésped y los factores ambientales, tales como el $\mathrm{pH}$ del suelo, determinan la estructura de la población rizobiana. Dentro de la gran diversidad de rizobios que existe, algunos son más específicos en cuanto a la elección del huésped, mientras que otros son más generalistas (Wei et al., 2007).

\section{Características del género Rhizobium.}

El género Rhizobium se caracteriza por ser bacterias móviles, Gram negativas y fácilmente cultivables. Requieren manitol como fuente de carbono y generalmente crecen a temperaturas entre 25 y $30{ }^{\circ} \mathrm{C}$. Actualmente muchas cepas de rizobios ocupan un lugar importante en la agricultura y son utilizadas como inoculantes, ya que mejoran en gran medida la FBN en condiciones adversas. Este género se destaca por su tolerancia a distintos tipos de estrés, como estrés salino, presencia de metales pesados en el suelo y estrés osmótico (Carevic, 
2014). La infección de los rizobios en las raíces de las leguminosas puede ocurrir a través de diferentes mecanismos: por los pelos radicales mediante la formación de "hilos de infección", a través de heridas o lesiones (crack entry) causadas por la emergencia de las raíces laterales o directamente por la epidermis. Como resultado de la infección se desarrollan los nódulos radicales, los cuales pueden tener distinta morfología y estructura (Sprent y Parsons, 2010).

\section{FBN en ecosistemas desérticos.}

La FBN tiene un gran impacto en la agronomía, economía y ecología debido a que la disponibilidad de $\mathrm{N}$ en el suelo limita la producción agrícola en todo el mundo. Además, este proceso es de suma importancia cuando se pretende transformar una región árida o semiárida en ecosistema productivo y sustentable, ya que entre los factores que aparecen como adversos en estas áreas se destacan los suelos pobres en N. Por lo tanto, la simbiosis con los rizobios favorece la fijación de $\mathrm{N}$ en zonas donde su deposición es muy baja y resulta una de las principales fuentes de ingreso al ecosistema árido (Wei et al., 2007).

En estas zonas se debe intentar cultivar las leguminosas nativas, ya que los microorganismos con los cuales se asocian se encuentran adaptados al ambiente y pueden establecer la simbiosis bajo condiciones de estrés, a diferencia de los que se inoculan en cultivos comerciales. Asimismo, las leguminosas nativas son candidatas ideales para su uso en proyectos de recuperación de tierras, ya que son resistentes a la sequía, mejoran la fertilidad del suelo y exhiben una fotosíntesis neta alta que aumenta su ganancia de carbono. Estas especies también cumplen funciones ecológicas importantes, como contribución de proteínas a la dieta de herbívoros, reducción de erosión del suelo, mejoramiento de la estructura y calidad del suelo, mejoramiento de la biodiversidad y de la polinización, aumentando de este modo la rentabilidad agrícola (Wei et al., 2007; Sprent y Parsons, 2000; Carevic, 2014). En la Figura 1, se muestra un mapa conceptual como recurso esquemático para representar un conjunto de significados abordados anteriormente, relacionando el proceso de desertificación con las leguminosas nativas. Esta figura se propone para mejorar la comprensión del texto y a modo de integración.

\section{Estudios de leguminosas nativas en Argentina}

A nivel mundial, se han realizado numerosos estudios de leguminosas nativas que habitan en ecosistemas áridos y semiáridos y que son capaces de establecer simbiosis con los microorganismos de suelo. En Argentina se han descrito pocas especies, y entre los géneros más estudiados se destacan Adesmia (Bianco et al., 2013), Desmanthus (Fornasero et al., 2014), Desmodium (Toniutti et al., 2017), Macroptilium (Toniutti et al., 2015) y Prosopis (Villagra et al., 2010).

\section{Género Adesmia.}

El género Adesmia (subfamilia Papilionoideae) posee cerca de 240 especies herbáceas o arbustivas que habitan en zonas montañosas y semidesérticas. Su distribución abarca desde el norte de Perú hasta Tierra del Fuego, y comprende Bolivia Occidental, Chile, Argentina, Sur de Brasil y Uruguay. En Argentina viven aproximadamente 100 especies y 14 variedades, de las cuales 53 son endémicas. $\mathrm{La}$ mayoría de las especies de Adesmia pueden crecer bajo condiciones adversas, soportando largos periodos de sequía y temperaturas extremadamente bajas (Burkart, 1967; Bianco et al., 2013). En Argentina se destaca Adesmia bicolor, la cual presenta potencial como forrajera debido a que persiste bajo pastoreo intensivo y es palatable para el ganado. Además tiene elevada biomasa, elevado contenido de $\mathrm{N}$ tanto en la parte aérea como subterránea, abundante nodulación y alta capacidad para la FBN. Adesmia bicolor es nodulada por bacterias que pertenecen al género Rhizobium y los nódulos son tipo aeschynomenoide (Bianco et al., 2013).

\section{Género Desmanthus.}

El género Desmanthus (subfamilia Mimosoidae) incluye cerca de 24 especies nativas de América. En Argentina se encuentran especies como Desmanthus paspalaceus, Desmanthus acuminatus, Desmanthus virgatus y Desmanthus tatuhyensis. Las tres primeras presentan potencial como forrajeras y todas son capaces de tolerar condiciones de sequía y de ser buenas competidoras frente a otras especies con las cuales conviven. Por ello se consideran de gran importancia como fuente de alimento para el ganado en regiones semiáridas del norte central y noroeste de Argentina (Fornasero et al., 2014). A nivel mundial, numerosas investigaciones se han centrado en el aislamiento, análisis y caracterización simbiótica de las cepas rizobianas capaces de asociarse con Desmanthus. En América del Sur, particularmente en Argentina, las especies estudiadas son Desmanthus paspalaceus y Desmanthus virgatus (Pedraza et al., 


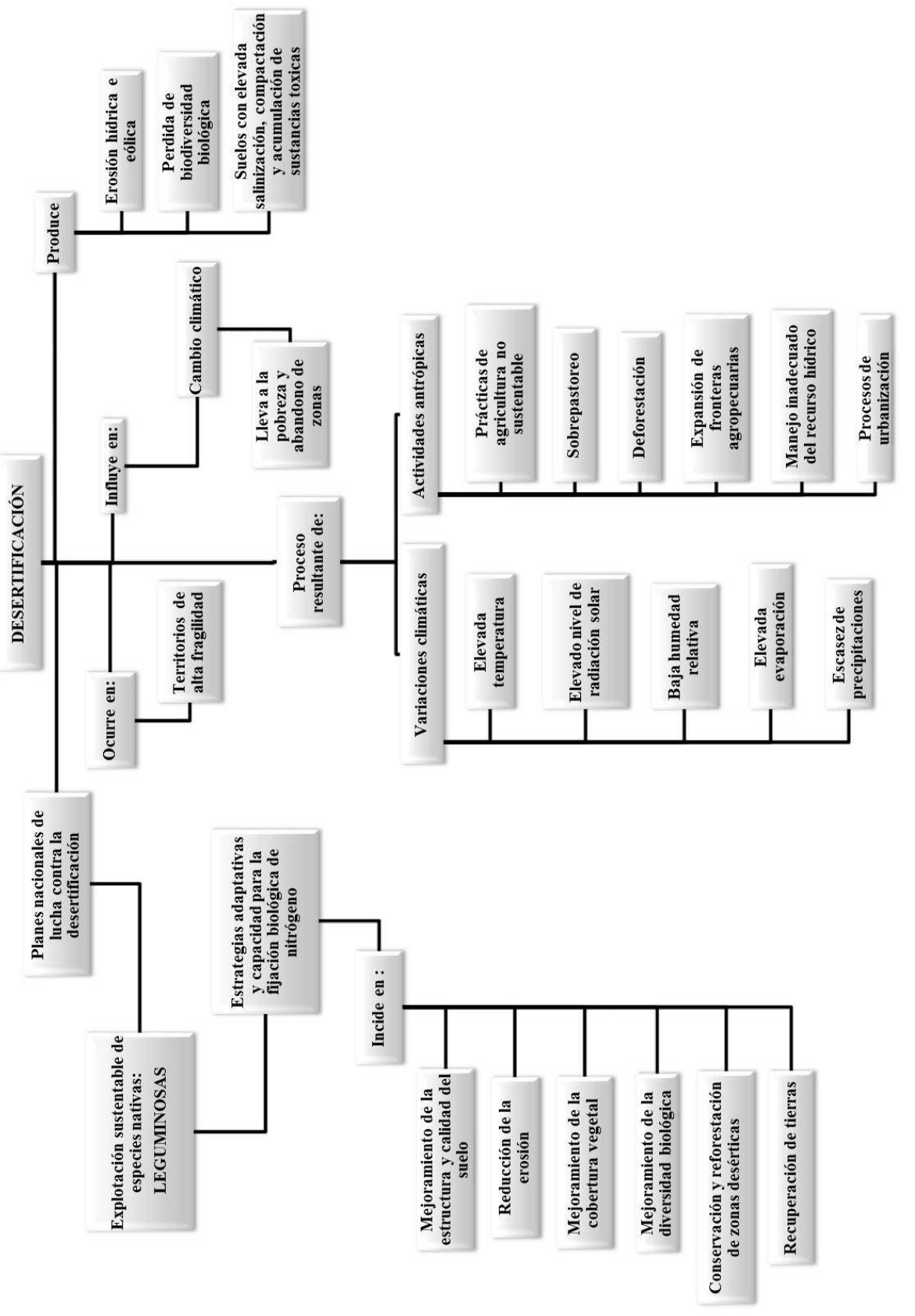

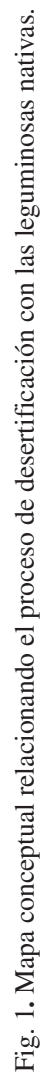


2001; Fornasero et al., 2014). Estudios realizados por Fornasero et al. (2014) en Desmanthus paspalaceus reportaron que esta especie es capaz de establecer simbiosis con una gran diversidad de bacterias de los géneros Rhizobium, Sinorhizobium y Mesorhizobium, y que los simbiontes asociados presentan tolerancia a condiciones de estrés abiótico como acidez, salinidad $\mathrm{y}$ altas temperaturas.

\section{Género Desmodium.}

ElgéneroDesmodium(subfamiliaPapilionoideae) está ampliamente distribuido en regiones templadas y subtropicales. El género incluye especies pioneras que resisten ambientes xerotérmicos y crecen en sitios áridos. En Argentina se han encontrado 23 especies nativas que son capaces de establecer simbiosis y fijar $\mathrm{N}$ atmosférico con los rizobios del suelo. En especies como Desmodium incanum se obtuvieron un total de 64 rizobios nodulantes pertenecientes a los géneros Bradyrhizobium y Rhizobium. Los rizobios mostraron una notable diversidad genética y tolerancia frente a distintas condiciones de estrés abiótico como altas temperaturas, $\mathrm{pH}$ extremo y salinidad, que son factores de estrés comúnmente encontrados en los suelos argentinos (Toniutti et al., 2017).

\section{Género Macroptilium.}

El género Macroptilium (subfamilia Papilionoideae) es nodulado por diversas bacterias como Bradyrhizobium sp. aisladas de leguminosas de los géneros Glycine, Vigna, Lupinus, Ornithopus, Cicer, Sesbania, Leucaena, Mimosa, Lablab y Acacia. También puede ser nodulado por cepas de Rhizobium y Ensinfer (Pedraza et al., 2001). En estudios realizados en Macroptilium atropurpureum y Macroptilium bracteatum se identificaron 7 aislados nativos capaces de crecer en condiciones de estrés como altas temperaturas, alcalinidad y salinidad. Macroptilium bracteatum es una especie nativa de América y en Argentina se encuentra en las regiones fitogeográficas Paranaense, Yungas y Chaqueña (Toniutti et al., 2015).

\section{Género Prosopis.}

El género Prosopis (subfamilia Mimosoideae) comprende árboles y arbustos, y se distribuye en distintas regiones de todo el mundo (Carevic,
2014). Se ha observado que su distribución se fue expandiendo sobre ambientes cada vez más xéricos y fríos. El proceso evolutivo se evidencia en las bioformas, espinas, hojas y frutos. Argentina es considerada un centro de diversidad mundial del género, ya que en sus ambientes se encuentran distribuidas unas 28 especies, de las cuales 13 son endémicas (Burkart, 1976). Las especies de Prosopis representan un recurso natural con características de interés. Sus adaptaciones les permiten crecer en suelos pobres de nutrientes donde pocas especies pueden sobrevivir. Además tienen una naturaleza polivalente, con el potencial de proporcionar un amplio rango de productos a la sociedad. Asimismo, muchas especies cumplen un papel muy importante en el proceso de desertificación, ya que reducen la erosión del suelo y aumentan la fertilidad mediante la producción de hojarasca. Otro aspecto significativo es que debido a su capacidad de colonizar y sobrevivir en ambientes marginales son utilizadas en programas de reforestación para las áreas degradadas (Carevic, 2014).

El género Prosopis presenta varias adaptaciones morfológicas y fisiológicas que les permiten a sus especies tolerar la sequía y así ocupar diferentes nichos ecológicos. Entre ellas podemos mencionar: I) Plasticidad en la arquitectura de sus raíces, lo que le permite utilizar tanto el agua superficial como subterránea, logrando de esta manera una independencia relativa de la variación en la disponibilidad de agua de las capas más superficiales del suelo, II) Cierre de estomas en ciertos momentos del día para evitar altas tasas de pérdida de agua por transpiración, III) Cierre de foliolos como mecanismo de fotoprotección frente a un exceso de radiación solar en condiciones de baja disponibilidad de agua, IV) Variaciones en la anatomía del leño a lo largo del desarrollo de la planta en ambientes con distinto régimen de precipitaciones, $\mathrm{y} \mathrm{V}$ ) Metabolismo $\mathrm{C} 3$ que le permite tener distintas eficiencias en el uso del agua y tolerancia a la temperatura (Burkart, 1976; Villagra et al., 2010; Carevic, 2014).

Las especies de Prosopis pueden ser noduladas por diversos tipos de rizobios como Mesorhizobium chacoense, Mesorhizobium plurifarium, Rhizobium etli, Rhizobium leguminosarum, Sinorhizobium arboris, Sinorhizobium meliloti, Sinorhizobium saheli, Sinorhizobium kostiense, Bradyrhizobium sp. y Ensifer sp. Los nódulos generalmente son de crecimiento indeterminado, a veces, con ramificaciones (Iglesias et al., 2007). 
Prosopis argentina y Prosopis alpataco son especies que están adaptadas a vivir en zonas de extrema aridez. Se distribuyen en la Provincia fitogeográfica del Monte, que se caracteriza por ser una de las zonas más áridas de la Argentina, con precipitaciones impredecibles, que varían entre 80 y $200 \mathrm{~mm}$ anuales y temperaturas medias de $15^{\circ} \mathrm{C}$ a 17 ${ }^{\circ} \mathrm{C}$ (Fernández y Busso, 1999). Prosopis argentina crece en suelos arenosos, mientras que Prosopis alpataco en suelos arcillosos, salinos y periódicamente inundados. Además de tener gran capacidad de adaptación, ambas poseen capacidad para fijar $\mathrm{N}$ atmosférico en simbiosis con los microorganismos del suelo. Otras especies del género con capacidad de establecer simbiosis y que están presentes en Argentina son Prosopis alba, Prosopis chilensis, Prosopis globosum y Prosopis flexuosa (Villagra et al., 2010).

\section{Conclusión}

Las leguminosas nativas son importantes desde el punto de vista ecológico y económico. Se destacan por presentar diversas características morfofisiológicas frente a diferentes tipos de estrés y por su capacidad para la FBN. Estos atributos no solo les permiten sobrevivir en zonas áridas y semiáridas donde otras especies no pueden crecer ni establecerse, sino también mejorar la estructura y la calidad del suelo. Por otro lado, las leguminosas nativas y sus simbiontes representan un germoplasma único que puede adoptarse para la restauración de los ecosistemas degradados, en el mantenimiento de la biodiversidad y para mitigar los efectos del cambio climático.

Los trabajos publicados en la actualidad muestran grandes avances en cuanto a la taxonomía rizobiana. La mayoría de estas especies bacterianas se encuentran en la familia Rhizobiaceae, clase $\alpha$-Proteobacterias. Sin embargo, estudios realizados recientemente han demostrado que existen otros géneros además de estos. En algunos casos la diversidad de los rizobios está relacionada con la distribución geográfica de las leguminosas.

En los últimos años se han realizado importantes estudios de modificaciones morfoanatómicas a nivel del sistema radical. Entre ellas se destaca la formación de shovel roots y cluster roots, que les proporcionan a las especies tolerancia a distintos factores de estrés y cumplen un rol importante en la solubilización de nutrientes limitantes.

En Argentina, los géneros de leguminosas nativas más estudiados son Adesmia, Desmanthus, Desmodium, Macroptilium y Prosopis. Aún hay muchas especies con caracteres adaptativos y capacidad de establecer simbiosis con los microorganismos del suelo que no han sido investigadas. Por ello sería de gran importancia continuar con los estudios a fin de aplicar estrategias de manejo y conservación de especies de interés para prevenir el avance de la desertificación en regiones áridas y semiáridas de nuestro país.

\section{Literatura citada}

Abraham, E.M.

2002. Luchacontrala desertificación en las tierras secas de Argentina; el caso de Mendoza. El agua en Iberoamérica: de la escasez a la desertificación. CYTED XVII, Programa Iberoamericano de Ciencia y Tecnología para el desarrollo. Aprovechamiento y gestión de recursos hídricos. En: Fernández Cirelli, A. y Abraham, E. (Eds.). Publ. CYTED XVII y CETA. Pp. 27-44.

Bianco, L.; Angelini, J.; Fabra, A.; Malpassi, R.

2013. Diversity and symbiotic efficiency of indigenous rhizobia-nodulating Adesmia bicolor in soils of central Argentina. Current Microbiology, 66:174-184.

Burkart, A.

1967. Sinopsis del género sudamericano de Leguminosas Adesmia DC. Darwiniana, 14: 463-586.

Burkart, A.

1976. A monograph of the genus Prosopis (Leguminosae subfam. Mimosoideae): catalogue of the recognized species of Prosopis. Journal of the Arnold Arboretum, 57:450-525.
Carevic, F.S.

2014. The role of ecophysiological studies in the genus Prosopis: implications for the conservation of droughtprone species. IDESIA, 32:77-81.

Cenzano, A.M.; Varela, M.C.; Luna, M.V.

2016. Ecophysiology of native species from Patagonian Monte, Argentina. In: Khan, M.A.; Böer, B.; Clüsener-Godt, M.; Öztürk, M.; Gul, B. (Eds.). Sabkha Ecosystems. Volume V: The Americas (Tasks for Vegetation Science). Springer. Switzerland. Pp. 369-387.

Delgado-Baquerizo, M.; Maestre-Gil, F.T.; GallardoCorrea, A.

2013. Biological soil crusts increase the resistance of soil nitrogen dynamics to changes in temperatures in a semiarid ecosystem. Plant and Soil, 366: 35-47.

Fernández, O.A.; Busso, C.A.

1999. Arid and semi-arid rangelands: two thirds of Argentina. Rala Report, 200:41-60. 
Fornasero, L.V.; Del Papa, M.F.; López, J.L.; Albicoro, F.J.; Zabala, J.M.; Toniutti, M.A.; Pensiero, J.F.; Lagares, A.

2014. Phenotypic, Molecular and Symbiotic Characterization of the Rhizobial Symbionts of Desmanthus paspalaceus (Lindm.) Burkart That Grow in the Province of Santa Fe, Argentina. PLoS ONE, 9:104636.

Gehlot, H.S.; Panwar, D.; Tak, N.; Tak ,A.; Sankhla, I.S.; Poonar, N.; Parihar, R.; Shekhawat, N.S.; Kumar, M.; Tiwari,

R.; Ardley, J.; James, E.K.; Sprent, J.I..

2012. Nodulation of legumes from the Thar desert of India and molecular characterization of their rhizobia. Plant Soil, 357: 227-243.

Granados-Sánchez, D.; Hernández-García, M.A.; Vázquez-

Alarcón, A.; Ruiz-Puga, P.

2012. Los procesos de desertificación y las regiones áridas. Revista Chapingo Serie Ciencias Forestales y del Ambiente, 19: 46-66

Iglesias, O.; Rivas, R.; García-Fraile, P.; Abril, A.; Mateos, P.;

Martínez- Molina, E.; Velázquez, E.

2007. Genetic characterization of fast-growing rhizobia able to nodulate Prosopis alba in North Spain. FEMS Microbiology Letters, 277: 210-216.

Levitt, J.

1980. Responses of plants to environmental stresses. Vol. II. Water, radiation, salt and other stresses. Academic Press. New York, U.S. 607 p.

Lewis, G.P.; Schrire, B.; Mackinder, B.; Lock, M.

2005. Legumes of the world. The Royal Botanic Gardens. Kew, Reino Unido. 577 p.

Pedraza, R.O; Roncedo, C.S.; de Bellone, S.C.; Bellone, C.H.; Pérez, H.E.

2001. Diferenciación de rizobios nativos que nodulan cuatro leguminosas forrajeras en Tucumán, Argentina. Ciencia del suelo, 19:79-81.

Pütz, N.

1996. Development and functions of contractile roots. In: Waisel, Y.; Eshel, A.; Kafkafi U. (Eds). Plants roots. The Hidden Half. Marcel Dekker. New York, US. Pp 859-874.
Raab, N.; Meza, F.J.; Franck, N.; Bambach, N.

2015. Empirical stomatal conductance models reveal that the isohydric behavior of an Acacia caven Mediterranean Savannah scales from leaf to ecosystem. Agricultural and Forest Meteorology, 213: 203-216.

Sprent, J.I.; Parsons, R.

2000. Nitrogen fixation in legume and non-legume trees. Field Crops Research, 65: 183-196

Sultan, S.E.

2003. Phenotypic plasticity in plants: a case study in ecological development. Evolution and Development, 5:25-33.

Tola, E., Henríquez-Saba, J.L., Polone, E., Dazzo, F.B., Concheri, G., Casella, S. and Squartini, A.

2009. Shovel roots: a unique stress-avoiding developmental strategy of the legume plant Hedysarum coronarium $\mathrm{L}$. Plant Soil, 322: 25-37.

Toniutti, M. A.; Fornasero, L.V.; Trod, B.S.;Zuber, N.E.; Córdoba, M.S. 2015. Caracterización fenotípica y funcional de rizobios noduladores de dos especies del Género Macroptilium. Revista FAVE - Ciencias Agrarias, 14:106-117.

Toniutti, M.A.; Fornasero, L.V.; Albicoro, F.J.; Martini, M.C.; Draghi, W.; Álvarez, F.; Lagares, A.; Pensiero, J.F; Del Papa, M.F. 2017 Nitrogen-fixing rhizobial strains isolated from Desmodium incanum DC in Argentina: Phylogeny, biodiversity and symbiotic ability. Systematic and Applied Microbiology, 40: 297-307.

Villagra, P.E.; Vilela, A.; Giordano, C.; Álvarez, J.A.

2010. Ecophysiology of Prosopis species from the arid lands of Argentina: what do we know about adaptation to stressful environments? In: Ramawat, K.G. (Ed.). Desert Plants. Springer. Berlin, Germany. Pp. 321-340.

Wei, G.H.; Zhang, Z.X.; Chen, C.; Chen, W.M.; Ju, W.T.

2007. Phenotypic and genetic diversity of rhizobia isolated from nodules of the legume genera Astragalus, Lespedeza and Hedysarum in northwestern China. Microbiological Research, 163: 651-662.

Weir, B.S.

2016. The current taxonomy of rhizobia. NZ Disponible en:. https:// www.rhizobia.co.nz/taxonomy/rhizobia. Consultado: 10/ene/2016. 